\title{
For better integration of the principles of agroecology in the specifications of French wine geographical indications / Pour une meilleure intégration des principes de l'agro-écologie dans les cahiers des charges des indications géographiques viticoles françaises
}

\author{
Jacques Gautier \\ INAO (Institut national de l'Origine et de la Qualité), France
}

\begin{abstract}
The French wine production is mainly produced under Geographical Indication (GI), with 378 PDO and 74 PGI wine, spread over its entire territory.

The concept of AOC, set up un 1936 by french Authorities, is entrusted to the INAO, public organization whose one of the missions is to validate the proposals of conditions of production developed jointly by producers within structures called organizations of defense and management. Compliance with these conditions is required to develop these products with a strong and not reproducible link with their terroir.

However it appears difficult to develop these wines without worrying about the preservation of this terroir, which is the main tool for producers.

This concern, which seems at present more and more obvious, however, was difficult to accept until recent years by the various components of the governance of the AOC, the producers, but also in some cases the public authorities.

A first reflection to the integration of agri-environmental measures in the rules of AOC started in the early 2000s, and was suspended without being able to lead to significant changes in conditions of production contained in the rules of AOC.

With the challenge of societal expectations and particularly the pressure of environmental expectations, the INAO has defined early 2016 innovative agro-ecological targets for all official signs of quality and origin, including wine geographical indications, inviting producers to move towards the ambitious guidelines that will apply to all producers of the GI.

For the wine sector 5 main themes were selected by the INAO:

- The preservation and development of biodiversity,

- The control of fertilization and in particular mineral nitrogen fertilization,

- The reducing the use of pesticides in particular by developing the use of bio-control,

- Looking for a better water management by operators,

- Using a genetic most suited to the challenges of agro-ecology.

This list is not exhaustive and can be completed according to local situations.

For each theme a set of standard measures will be proposed by the INAO. The integration of these measures in the specifications will be almost automatic, if requested by the producers.

Factsheet of these measures will be provided to defense organizations and management of GIs.

Very soon, the ODG will have the opportunity to collectively define the measures they consider most appropriate, which will then be integrated into their specifications.

All the proposed measures will make a response to the challenge of the expectations of society, in particular to strengthen an environmental approach, to enhance the credibility of a system based on the link between the product and its terroir, while preserving the collective heritage of GI French wine.
\end{abstract}

La production viticole française est très majoritairement produite sous indication géographique (IG), avec 378 AOC (appellation d'origine contrôlée) et 74 IGP viticoles (indications géographiques protégées).
Ces indications géographiques, réparties sur la quasitotalité du territoire national, représentent en effet plus de $90 \%$ de la production viticole française, le solde de la production française étant commercialisé en vin sans indication géographique (VSIG). 


\section{Les indications géographiques, $A O C$ et IGP, sont des dénominations basées sur une qualité des produits liée à leur origine}

\subsection{Les produits bénéficiant d'une $A O C$ sont très fortement liés à leur terroir}

Lors de la mise en place en France du système des AOC viticoles en 1935, les Pouvoirs Publics français ont souhaité instaurer un système permettant une différenciation significative de ces produits d'AOC d'avec les produits courants rencontrés sur le marché.

Une différenciation significative contribue notamment à une meilleure rémunération des producteurs, le consentement à payer des consommateurs pour ce produit différent du produit standard étant significativement plus élevé.

Le système des AOC s'est appuyé sur des productions présentant préalablement une forte notoriété développée collectivement par les acteurs locaux, et dont les qualités substantielles des produits étaient liées à un terroir délimité précisément.

Au vu de la multiplication des définitions retenues pour le « terroir », amenant par voie de conséquence une très forte 'hétérogénéité des « produits de terroir »s'y référant, il a été nécessaire de retenir au niveau international une définition du terroir.

La définition du terroir viticole votée par l'OIV en 2010 a permis de se référer à une définition unique : « Le « terroir »vitivinicole est un concept qui se réfère à un espace sur lequel se développe un savoir collectif des interactions entre un milieu physique et biologique identifiable et les pratiques vitivinicoles appliquées, qui confèrent des caractéristiques distinctives aux produits originaires de cet espace. Le « terroir $»$ inclut des caractéristiques spécifiques du sol, de la topographie, du climat, du paysage et de la biodiversité. 》

Il est important de rappeler que si bien évidemment les caractéristiques du milieu naturel (sol, climat notamment) ont une contribution essentielle dans un terroir, la notion de terroir comprend également les pratiques vitivinicoles mises en œuvre durant l'ensemble du cycle de production par les opérateurs.

La mise en oeuvre des pratiques culturales inscrites dans les dispositions du cahier des charges de chaque AOC permet au produit d'acquérir les caractéristiques fondamentales de l'AOC.

\subsection{Les produits bénéficiant d'une indication géographique protégée (IGP) présentent également des caractéristiques particulières liées à leur origine géographique}

La définition des IGP s'applique à des produits originaires d'une aire géographique de production définie et dont une qualité déterminée, la réputation ou d'autres caractéristiques particulières peuvent être attribuées à leur origine géographique.

En 2010 les travaux de reconnaissance des IGP françaises se sont largement appuyés sur la catégorie des vins de table commercialisés préalablement en « vins de pays », et qui répondaient à la définition des IGP.
L'organisation existant pour les AOC a été reconduite pour les IGP, avec le même système de cogestion existant entre les opérateurs (également regroupés dans une structure collective dénommée organisme de défense et de gestion), les Pouvoirs Publics français et l'INAO.

En 201674 IGP françaises viticoles ont été reconnues, représentant environ $20 \%$ de la production française.

\subsection{Un système original de cogestion pour les indications géographiques, AOC et IGP}

Depuis la création du système des AOC en 1935, la gestion des indications géographiques a été confiée à l'INAO, organisme public dont une des missions est de valider les propositions de condition de production élaborées collectivement par les producteurs regroupés dans des structures collectives. Longtemps dénommées syndicat de défense de l'appellation, ces structures sont dorénavant reconnues par l'INAO comme étant les organismes de défense et de gestion (ODG) des indications géographiques.

Les éventuelles propositions d'évolution des dispositions contenues dans les cahiers des charges sont donc de la seule compétence de l'ODG.

Une fois le dossier transmis à l'INAO, l'examen et la validation des propositions des ODG est assurée par le comité national des appellations d'origine, structure majoritairement composée d'acteurs professionnels (notamment producteurs et négociants) de la filière viticole française concernée, AOC ou IGP.

Les décisions prises par le comité national de l'INAO sont ensuite transmises au Ministère français de l'Agriculture, pour publication du cahier des charges de l'AOC au Journal Officiel. Par la suite interviendra la procédure d'enregistrement auprès des autorités communautaires.

Le respect de l'ensemble des conditions de production est une condition nécessaire pour bénéficier de l'IG, contribuant à l'obtention d'un produit présentant les caractéristiques spécifiques ayant permis la reconnaissance de l'indication géographique par les Pouvoirs Publics.

Ce lien des produits avec leur terroir, issu du milieu naturel et des pratiques mises en oeuvre, ne peut être reproduit hors de la zone de production.

Le système de cogestion mis en place depuis 80 ans pour les AOC a associé les producteurs (via leur ODG), les Pouvoirs Publics et l'INAO. Le pouvoir de proposition dans l'établissement des conditions de production de leur AOC est de la seule responsabilité des ODG : qui connait le mieux les règles de production vitivinicoles permettant d'aboutir à un produit d'exception, si ce n'est les producteurs eux-mêmes ?

Ce même système a été étendu aux IGP viticoles depuis leur mise en place en 2010.

\section{Vers une nécessaire évolution des conditions de production des indications géographiques}

L'élaboration de vins liés à leur terroir d'origine sousentend en particulier une préoccupation permanente préserver ce terroir, qui constitue l'outil de travail principal des producteurs.

Cette préoccupation, qui parait à l'heure actuelle de plus en plus évidente, a toutefois été difficile à 
faire accepter jusqu'à ces dernières années par les différentes composantes de la gouvernance des indications géographiques, producteurs mais également dans certains cas Pouvoirs Publics.

Ainsi une première réflexion visant à l'intégration des mesures agro-environnementales dans les règles de production des AOC engagée au début des années 2000, avait été suspendue sans avoir pu se traduire par des évolutions significatives des conditions de production contenues dans les cahiers des charges des AOC.

Durant la dernière décennie 2005/2015 les attentes sociétales ont considérablement évoluées, et se traduisent notamment par une recherche de produits de plus en plus respectueux de l'environnement.

Début 2016, suite à la levée d'un certain nombre d'interrogations réglementaires par le Ministre de l'Agriculture, l'INAO a invité les ODG à s'orienter clairement et fermement vers une démarche d'intégration des principes agro-écologiques dans les cahiers des charges de l'ensemble des signes officiels de qualité et d'origine.

Cette orientation répondait à une revendication déjà ancienne des ODG de pouvoir intégrer de telles dispositions environnementales dans les cahiers des charges.

Cette orientation est ambitieuse, puisqu'elle s'appliquera à l'ensemble des producteurs de l'indication géographique concernée et ce dès l'incorporation des mesures dans les cahiers des charges. Elles devront toutefois, pour pouvoir être acceptées et mises en oeuvre par l'ensemble des producteurs, faire l'objet d'un rythme adapté à chaque IG, qui tiendra compte des différentes situations.

Cinq thématiques ont prioritairement été retenues pour les productions végétales et la production viticole :

- la préservation et le développement de la biodiversité,

- la maitrise de la fertilisation, et notamment de la fertilisation azotée minérale,

- la réduction de l'usage des produits phytosanitaires notamment par le développement de l'usage du biocontrôle,

- la recherche d'une meilleure gestion de l'eau par les opérateurs,

- le recours à une génétique plus adaptée aux enjeux de l'agro-écologie.

Cette liste n'est pas exhaustive, et pourra être complétée par les ODG pour tenir compte des contextes particuliers spécifiques à chaque IG.

Pour ces cinq thématiques un catalogue de mesurestypes est en cours d'élaboration par l'INAO en concertation avec les instituts techniques de chaque filière. Ce catalogue, qui décrira les mesures par le biais de fiches descriptives, sera ensuite présenté aux comités nationaux pour approbation.

L'inscription de ces mesures au catalogue permettra leur intégration simplifiée et rapide dans les cahiers des charges, sous réserve de l'existence d'une demande déposée par l'ODG de l'IG concernée.

Avec d'autres méthodes de certification environnementales existant déjà par ailleurs (HVE, ...), les ODG et les opérateurs disposeront d'un ensemble élargi d'outils pour construire leurs ambitions agro-écologiques.

Chaque ODG pourra évoluer au rythme qu'il aura luimême défini avec l'ensemble des opérateurs, rythme qui tiendra compte des situations, des moyens, des pressions extérieures, .... Durant la mise en place de cette réflexion l'INAO a toujours précisé que l'intégration de critères agro-écologiques serait une démarche volontaire pilotée par chaque ODG.

Il serait toutefois paradoxal que les indications géographiques françaises et notamment celles du secteur viticole, qui ont régulièrement démontré être à la pointe de l'innovation, ne soient pas à l'avant-garde de cette démarche. Dans le cas contraire elles risqueraient de se voir imposer de nouvelles normes environnementales par la distribution, voire d'être contraintes de respecter une nouvelle réglementation encore plus contraignante.

\section{En conclusion}

Dès la fin 2016 les ODG auront l'opportunité de retenir collectivement les mesures qui leur paraissent les plus opportunes, qui seront alors intégrées dans leurs cahiers des charges.

L'ensemble de ces orientations va permettre d'apporter une réponse au défi des attentes sociétales, et notamment de conforter une approche éco-citoyenne et environnementale, tout en permettant de renforcer la crédibilité d'un système basé sur le lien fort et non reproductible $\mathrm{du}$ produit avec son terroir d'origine, et de préserver le patrimoine collectif que constituent les IG viticoles françaises.

\author{
Jacques GAUTIER \\ INAO \\ Inspecteur national \\ 1 Quai Wilson \\ 33 130 BEGLES - BORDEAUX \\ j.gautier@inao.gouv.fr
}

